\title{
Objective measure of woven fabric's cover factor by image processing
}

\begin{abstract}
Digital image processing techniques are applied to perform an automatic method for the objective measure of woven fabric's cover factor. Based on a frequency domain analysis, digital images of woven fabric samples, obtained with a camera assembled to a microscope, are cropped to enclose the maximum integer number of warp and weft periods and leveled for non-uniform illumination. Posterior thresholding, designed to perform suitably for both high and low cover factor fabric samples, gives rise to the objective value. The method has been applied to three different sets of samples manufactured in plain weave, with known yarn numbers and thread counts. Cover factors obtained by this method show good correlation with those obtained by a set of visual observers and are consistent with woven fabric parameters: yarn numbers (tex) and thread counts (yarns/cm). The procedure can be useful to monitor mean cover factor as well as cover factor variability in fabric batches. It does not require sophisticated equipment and can be straightforwardly implemented in a textile analysis laboratory.
\end{abstract}

Keywords - Image analysis, cover factor, woven fabrics

\section{Introduction}

Cover factor, $C F$, or overall cover factor is defined as the ratio of the area covered by yarns to the total area of the web [1].

$$
C F=\frac{\text { area covered by yarns }}{\text { total area of the web }}
$$

$C F$ is a basic construction parameter of woven cloth related to its end use performance [2,3]. Weaving efficiency, fabric quality [4,5], thermo-physiological comfort of garments, air permeability [6] as well as protection against Ultra Violet Radiation [7] are features strongly related to $C F$. It is a fundamental feature of multiple base fabrics used in the elaboration of 
protective garments and textiles designed to protect the working environment or the natural environment against the exposition to hazardous elements. They are found in common safety systems in cars like airbags, for instance. $C F$ is a critical parameter in protective clothing required in environments exposed to this kind of risks, ranging from pharmaceutical research laboratories to greenhouses affected by the risk of pesticides used in intensive agriculture. It is also a key factor in geotextiles to protect the environment from erosion or dangerous wastes or in garments for cleanrooms or surgery. The list would be endless and CF of base fabric plays a main role in the aforementioned examples. In addition to other textile parameters, achievement of an effective protection is not only attributable to the mean $C F$ value, but also to the uniformity of the $C F$ along the base fabric.

Mean $C F$ values of woven fabrics can be estimated from yarn numbers and thread counts by means of several well-known theoretical formulae $[8,9,10,11]$. Nevertheless, objective fabric inspection procedures should be established to monitor $C F$ mean value and its variability. Digital image processing techniques enable a more accurate and objective evaluation of $C F$ from small fabric areas than those resulting from theoretical formulae. Further statistical analysis of the $C F$ values obtained by suitably sampling across the fabric should provide estimates of the mean $C F$ value, as well as its variability.

Evaluation of CF from digital images of fabric samples has been considered in some previous works [12-15]. The procedure applied in [12] is briefly described in two steps: once the area corresponding to a single weave repeat was manually determined on a microscope image of a fabric sample, $C F$ of the fabric was obtained as the ratio of the area covered by the yarns to the total area of the weave repeat. Areas were evaluated with the help of a precision grid.

In [13-15] CF was straightforwardly derived by tallying the pixels in binarized images produced by thresholding. 
In [13] a fabric sample was illuminated by transmission and the threshold value defined as the average intensity of the background image without the fabric sample. So, it was related to the experimental settings. In [14] and [15] the fabric sample was illuminated by reflection and transmission. In [14] the threshold value was defined as the maximum intensity of the pixels corresponding to the lightest fiber, thus requiring some operator involvement. In [15] it was derived as a value separating two modes in the bimodal histogram of a digital fabric sample image, so it was only suitable for fabrics with low cover factor, displaying bimodal intensity histograms.

Besides accuracy and objectivity, automation is an important requirement for quality control procedures and the foregoing methods are difficult to implement in a fully automatic algorithm. The goal of this work is to develop an accurate, objective and automatic evaluation method of a fabric sample $C F$ based on a single digital image of the weave. The method would handle a wide range of $C F$ values, including those close to 1.

The proposed procedure for the automatic evaluation of the cover factor from microscope images of fabric samples illuminated by transmission consists of two steps. First we perform an analysis of structural features of a fabric sample from the power spectrum of its digital image. Our purpose is to determine the biggest region within the original image containing an integer number of complete periods, or thread interlacings. $C F$ values obtained from images containing a fractional number of thread interlacings would yield inaccurate or biased results. So, we crop the initial image to fit that region.

In a second step, once a digital image of a fabric sample has been cropped to contain an integer number of periods, fabric sample $C F$ is obtained from a classification of pixels into two classes: one corresponding to the threads - low intensity or dark pixels - and the other corresponding to the areas not covered by threads (holes) - high intensity or bright pixels -. Gray pixels, located close to thread edges, may be difficult to classify. Misclassification effects may produce 
relatively large departures from exact $C F$ for fabrics with high cover factor values. A correct classification of pixels by intensities requires a suitable thresholding procedure. Standard automatic thresholding methods [16] are not suited to unimodal histograms yielded by high $C F$ fabrics. We propose a thresholding procedure based on a slight modification of Otsu's thresholding method, applicable to fabrics whose respective intensity histograms are bimodal or unimodal shaped.

We apply this method to digital images of plain fabric samples with both low and high $C F$. Our interest focuses on high CF fabrics. To test the performance of the method we have considered a set of 81 fabric samples with known yarn numbers, thread counts and types of fiber. Their $C F$ values have been also evaluated according to an interactive thresholding procedure carried out by a group of observers and yielding $C F$ values approximately ranging from 0.90 to 1.00 ,

Validation arises from linear regressions comparing the mean $C F$ fitted by inspectors to the $C F$ yielded by the proposed method for each type of fiber. The results also show a strong relationship with the known fabric features as proved by an analysis of variance.

\section{Delimiting a Suitable Region by Fourier Analysis}

For the sake of simplicity we present the method to determine the biggest region containing an integer number of complete periods based on woven fabric models.

Since a woven fabric consists of regular repeating units, the Fourier Transform (FT) is particularly useful for analyzing periodicities and orientations in the fabric.

$F T$ of an image $f(x, y)$, in the spatial domain, is a two dimensional complex function $F(u, v)$, in

the frequency domain [17]. Its Power Spectrum $(P S),|F(u, v)|^{2}$, is a real function accounting for the distribution of the energy of the initial image over the spatial frequencies. It is worth noting that all PS in this work are displayed in logarithmic reversed scale (darkest pixels corresponding to highest intensities). 
The PS of a quasi-periodic image, such as a fabric sample image, is composed of peaks, aligned in a two dimensional array, whose value and location point out the frequency values and directionalities of the periodic structure.

Figure 1(a) displays a model of a perfectly periodic fabric sample with orthogonal warp and weft threads whose directions are oriented parallel to the coordinate axes. Figure 1(b) only contains its weft threads. Their corresponding PS are found in figures 2(a) and 2(b), respectively. Note that the highest intensity frequencies within the frame in figure 2(a) agree with the highest intensity frequencies in figure 2(b), concluding that the central column of the FT contains the information about the periodicity of the horizontal structure of the fabric image, i.e., the periodicity of weft yarns. The remaining frequency peaks in figure 2(a) are related to other features of the model fabric image $[18,19,20]$. In a similar way, the central row of the $F T$ accounts for the periodic structure of warp yarns.

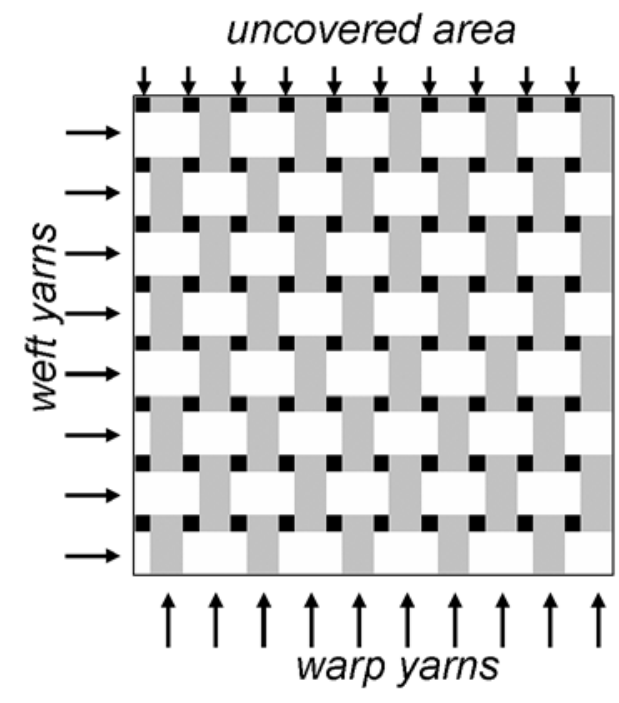

(a)

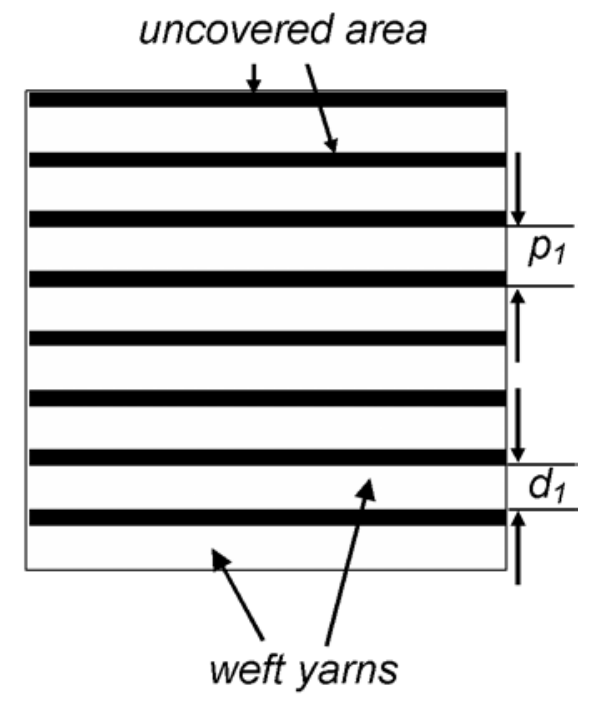

(b)

Figure 1 (a) Weave model. (b) Weft yarns, $d_{1}$ : Weft yarn diameter, $p_{1}$ : Weft length period. 


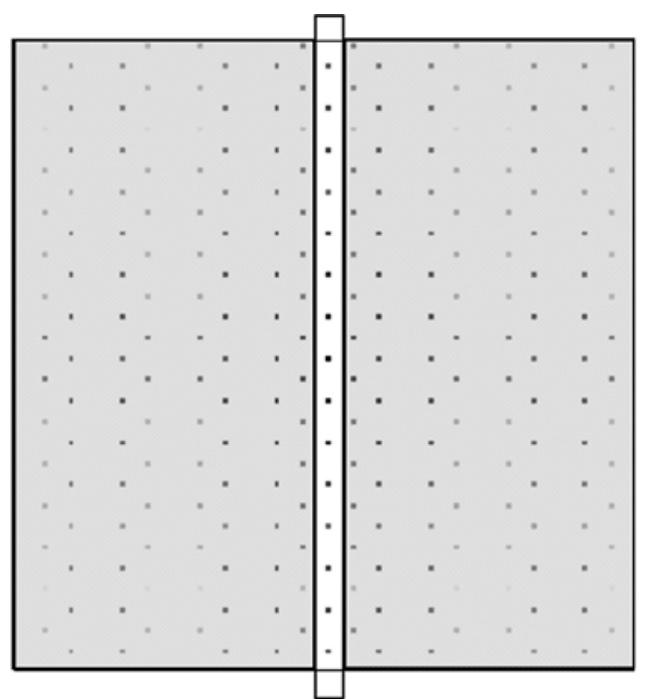

(a)

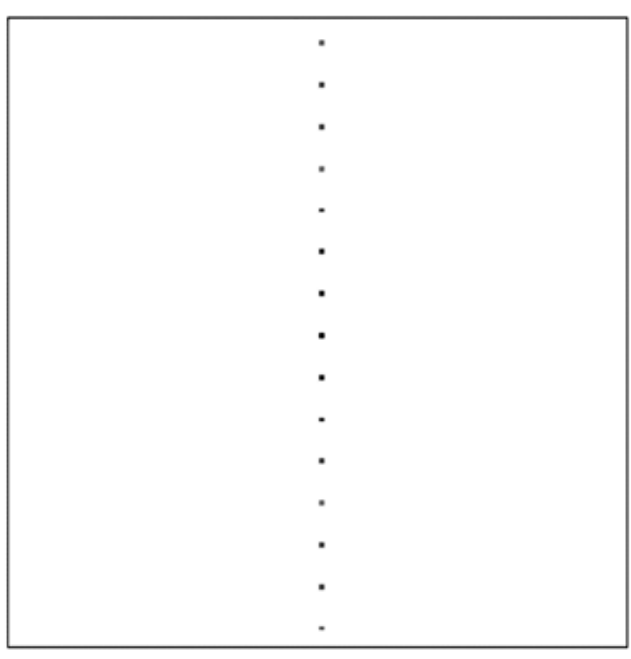

(b)

Figure 2 PS of figures 1(a) and 1(b) displaying the frequency match of the central column.

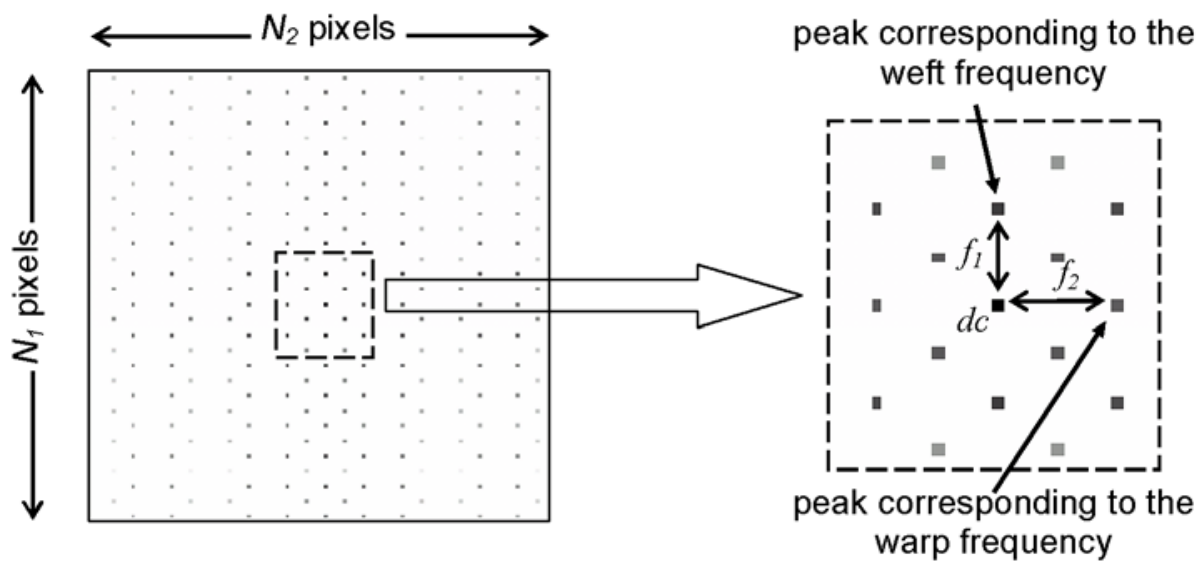

(a)

(b)

Figure 3 (a) PS of figure 1(a). (b) Enlarged image of figure 1(a): distances $f_{1}$ and $f_{2}$ correspond to weft and warp frequencies respectively.

Figure 1(a) contains an integer number of periods, consequently its $P S$ is a weighted array of Dirac deltas as shown in figure 3(a). Figure 3(b) displays an enlargement of figure 3(a). The distance $f_{1}$, in pixels, from the central peak of the $P S$, or $d c$ term, to the first peak in the vertical direction, coincides with the number of periods (weft threads) contained in the original image in the vertical direction $[18,19,20]$. Note that the $P S$ is symmetrical with respect to the $d c$ term. 
Count of warp threads $f_{2}$ follows analogously (Figure 3(b)). Their corresponding period lengths are

$$
p_{i}=\frac{N_{i}}{f_{i}}
$$

where $N_{i}$ is the number of pixels of the sample image in either direction $(i=1,2)$. The size of the whole image can be expressed as: $N_{1} \times N_{2}=f_{1} \cdot \frac{N_{1}}{f_{1}} \times f_{2} \cdot \frac{N_{2}}{f_{2}}=f_{1} \cdot p_{1} \times f_{2} \cdot p_{2}$.

When the number of periods in the sample image is not an integer, the energy of the PS is not concentrated on a perfect array of pixels, but slightly spread around them. In that case $f_{i}$ is obtained as the fractional distance between the $d c$ term and the center of mass corresponding to the intensities of the pixels around the first frequency peak on the PS in a direction orthogonal to the threads. The number of complete periods contained in the image in each direction are [ $f_{i}$ ] $(i=1,2)$, where [.] stands for the integer part. Recalling that period lengths are $p_{i}(i=1,2)$, the dimensions of the biggest region within the fabric sample image enclosing an integer number of periods in any direction are $N_{1}^{\prime} \times N_{2}^{\prime}=\left[f_{1}\right] \cdot p_{1} \times\left[f_{2}\right] \cdot p_{2}$. Thus, the initial image is cropped to fit the dimensions of this window or its closest integer dimensions.

\section{Thresholding Operation}

The segmentation algorithm requires some previous image pre-processing. Brightness of microscope images usually decays from the center to its borders. Image brightness and contrast are leveled by removing low-frequency terms in the Fourier space [21]. To this end, a ring shaped mask around the $d c$ term is applied to the FT of the original image and then backtransformed.

Computer screens enhance image contrast by a non-linear relationship between monitor's gray level and equivalent luminance [22, 23]. To match that behavior, a quadratic lookup table, assigning to each pixel its squared intensity, is applied to the previously leveled image. The resulting image is subsequently normalized to a 0-255 range for gray levels. 
Fabric samples were illuminated by transmission to minimize the effect of color and to increase contrast between threads and background. With this illumination, darker pixels correspond to threads and brighter pixels to uncovered areas. Standard automatic thresholding methods [16] failed to properly classify pixel intensities for some high $C F$ fabric samples. Based on the correspondence between pixels with highest intensity and the uncovered areas of the sample, we propose a slight modification of Otsu's method to threshold histograms coming from preprocessed fabric sample images. For every intensity $L$ between the minimum intensity and the maximum intensity of the sample image, we define the sum of squared residuals (SSQ) corresponding to $L$ by:

$$
\operatorname{SSQ}(L)=\sum_{i_{p} \leq L}\left(i_{p}-m(L)\right)^{2}+\sum_{i_{p}>L}\left(i_{p}-M\right)^{2}
$$

Where $i_{p}$ stands for the pixel's intensity, $m(L)$ is the average of all intensities lower than or equal to $L$ in the sample image and $M$ is the maximum intensity of the sample image. Note that $M$ substitutes the mean intensity of the pixels brighter than $L$ in Otsu's method. The first sum of equation 3 extends over all pixels with intensities lower than or equal to $L$ and it accounts for the sum of the squared residuals corresponding to the area covered by the yarns. The second sum extends over all pixels with intensities higher than $L$. It accounts for the squared residuals corresponding to the uncovered areas. The intensity $L_{0}$ that minimizes $S S Q$ is the threshold value.

$C F$ of the fabric sample is straightforwardly derived from the thresholded image as the ratio of pixels classified as corresponding to yarns over the total number of pixels.

\section{Application to real samples}

Fabric images were obtained with the experimental device illustrated in figure 4. All samples were unbiased or presented a small bias $\left(<5^{\circ}\right)$ and contained a number of warp threads and weft treads greater than 7. They were illuminated by transmission and a CCD camera (Olympus Camedia C-3030 Zoom), assembled to a microscope Olympus CX 40, yielded 24 bit color 
images, in TIFF format, $1536 \times 2048$ pixels in size $(2.6$ x $3.4 \mathrm{~mm}$ linear field size) with a 2x magnification. These images were converted to a 256 gray level scale and their resolution reduced 4 times (384x512 pixels) to shorten the processing time. A ring shaped mask around the dc term, with inner radius 1 and outer radius 3, is applied to the FT to level image brightness and contrast.

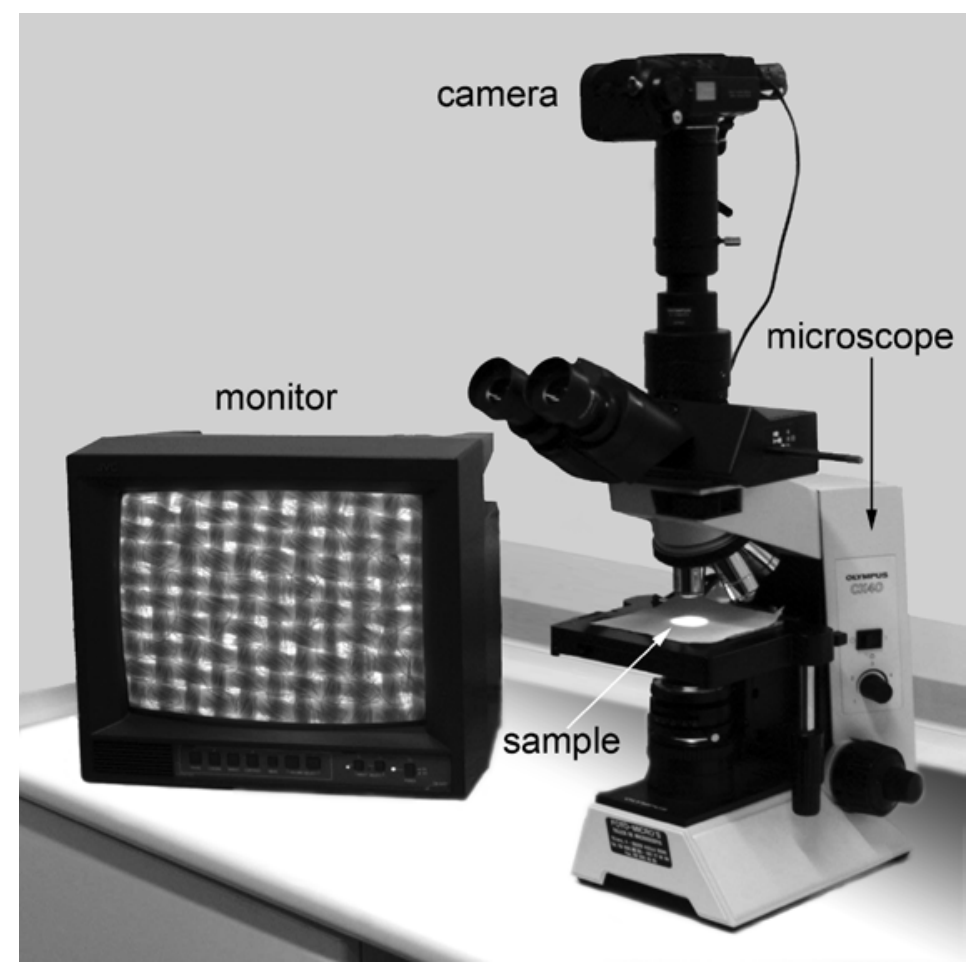

Figure 4 Experimental set up of imaging device.

The method is stepwise applied to actual digital images of two fabric samples. The sample displayed in figure 5(a) is a tightly woven fabric with high $C F$. Figure 6(a) displays a low $C F$ sample. Figures 5(b) and 6(b) show their corresponding PS.

The four highest peaks around the $d c$ term in 5(b) and 6(b) are the fundamental frequencies. Next, a detailed evaluation of $f_{1}$ and $f_{2}$ is performed for the first sample. Setting the coordinates of the $d c$ term to $(0,0)$ in figure $5(\mathrm{~b})$, the position of the peak at its right side is $(10,0)$ and the position of the peak above it is $(1,10)$. We consider squares with center at each peak and sides of length 7 , to separate each peak from its neighbors, and then find the centers of mass 
corresponding to each square. They are found to be $(0.80,9.71)$ and $(10.37,0.03)$. Distances of these centers to the $d c$ term are $f_{1}=9.71$ and $f_{2}=10.37$. Recall that these values coincide with the number of threads, or complete periods, in the warp and weft directions.

According to equation 2, the period lengths are $p_{1}=39.45$ pixels and $p_{2}=49.29$ pixels. In order to obtain an integer number of periods, the image $f(x, y)$ is cropped to $N^{\prime}{ }_{1}=355$ rows and $N{ }^{\prime}{ }_{2}=$ 493 columns, the rectangle plotted in figure 5(a). Similar arguments led to the rectangle plotted in figure 6(a).

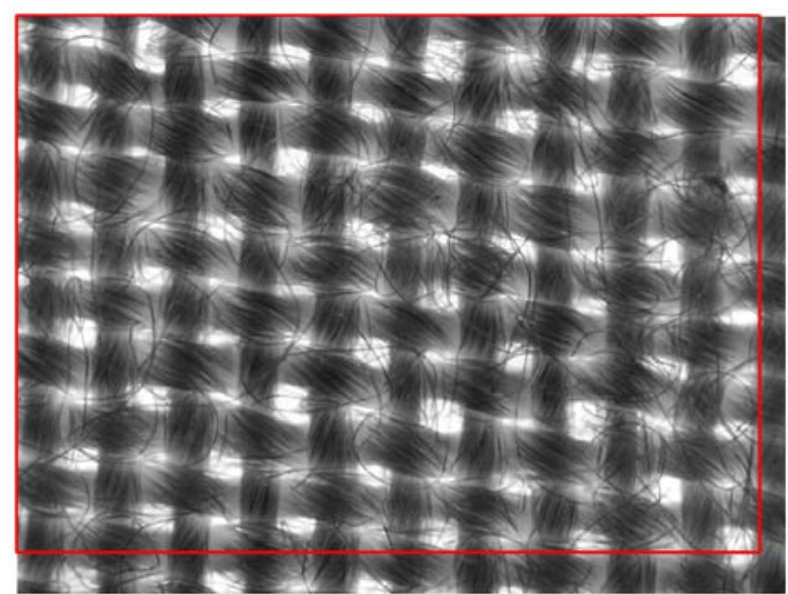

(a)

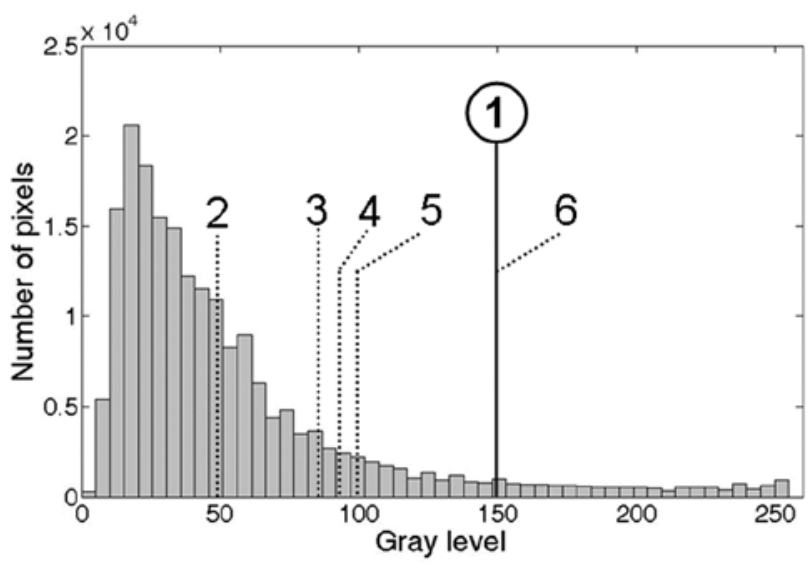

(c)

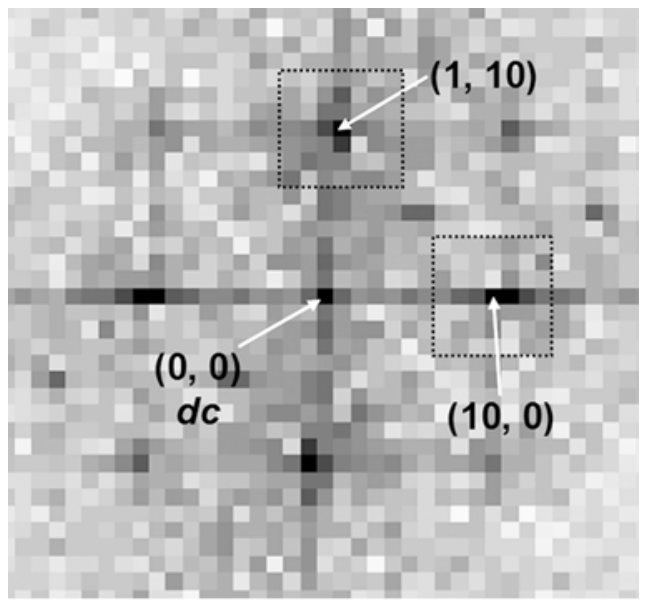

(b)

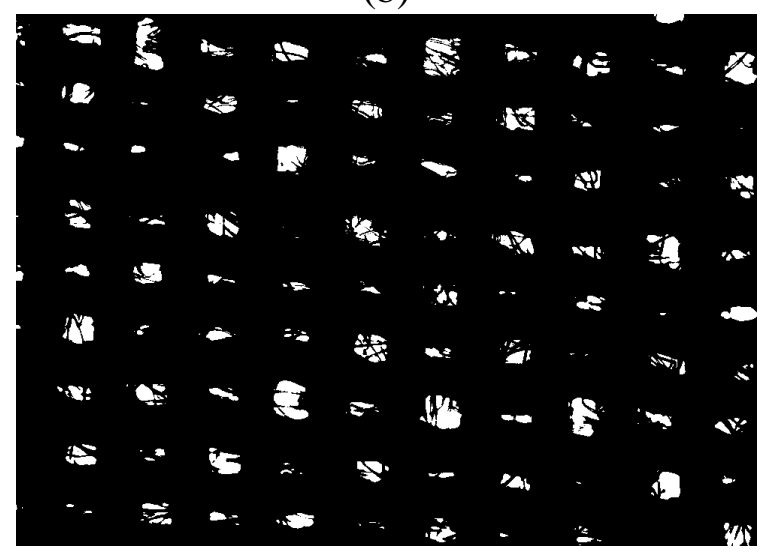

(d) 


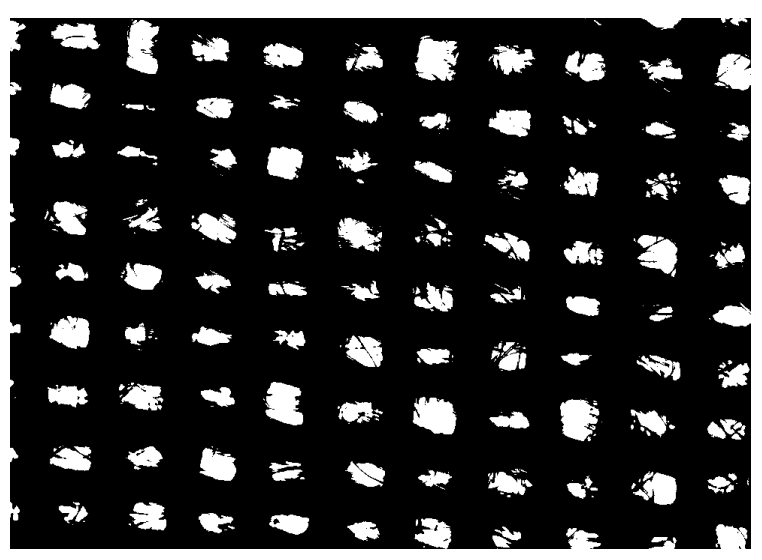

(e)

Figure 5 (a) High CF fabric with a rectangle containing an integer number of periods. (b) PS of 5(a) and squared areas enclosing warp and weft frequency peaks to evaluate the centers of mass. (c) Intensity histogram of the image delimited by the rectangle in 5(a) displaying threshold values yielded by different thresholding methods. 1: MSSQ, 2: GM, 3: K-I, 4: Otsu, 5: K-means and 6: Visual. (d) Binarization of the cropped image by MSSQ method. (e) Binarization of the cropped image by Otsu's method.

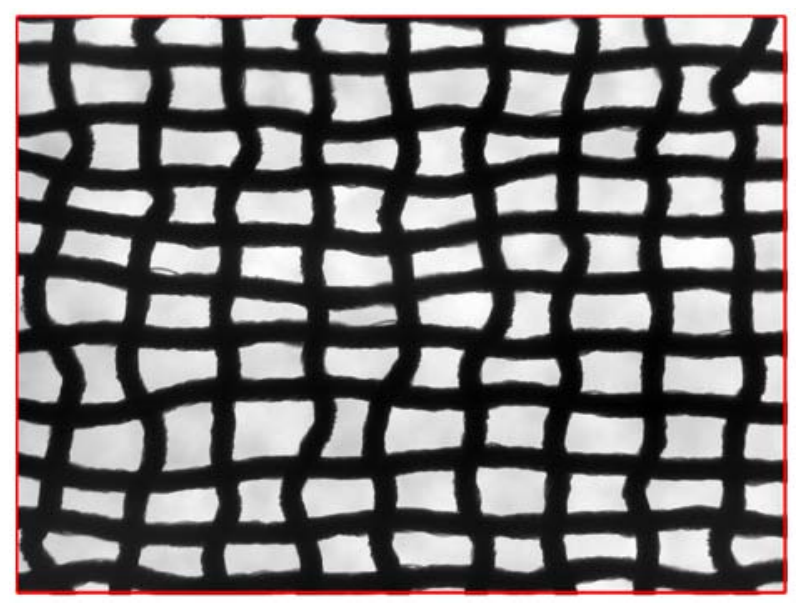

(a)

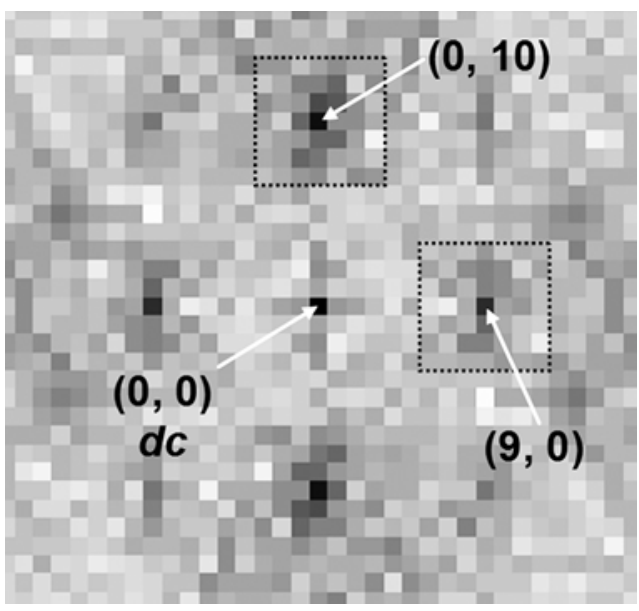

(b) 

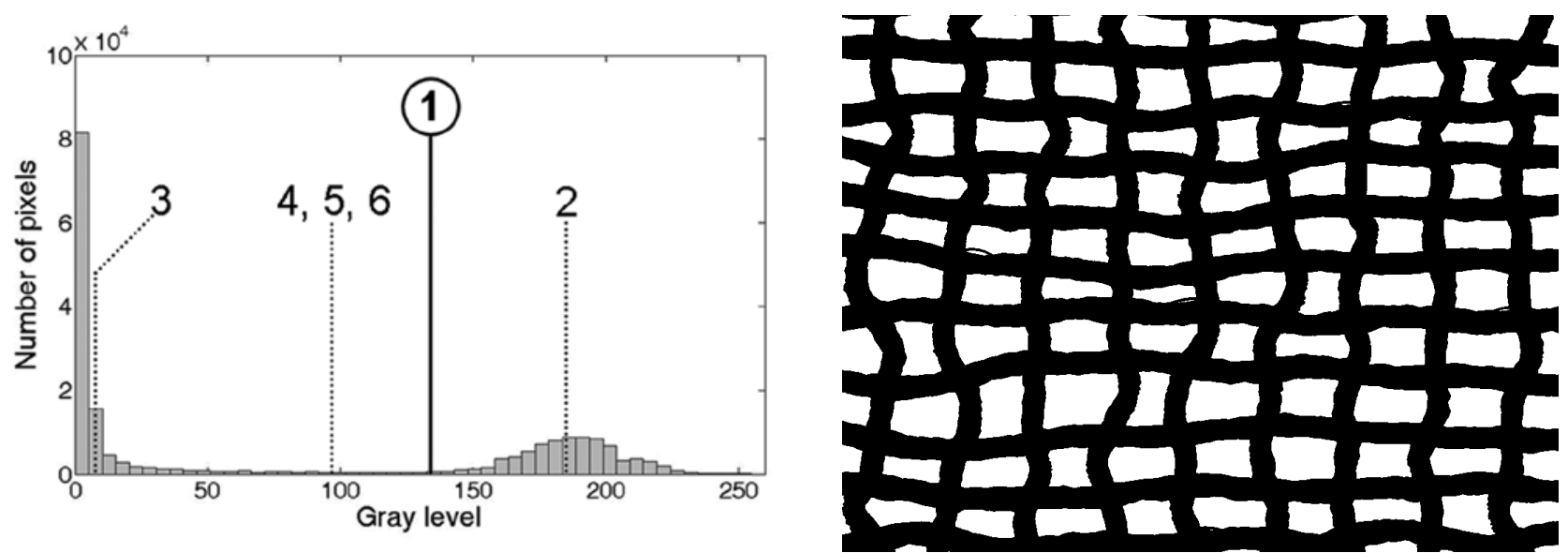

(c)

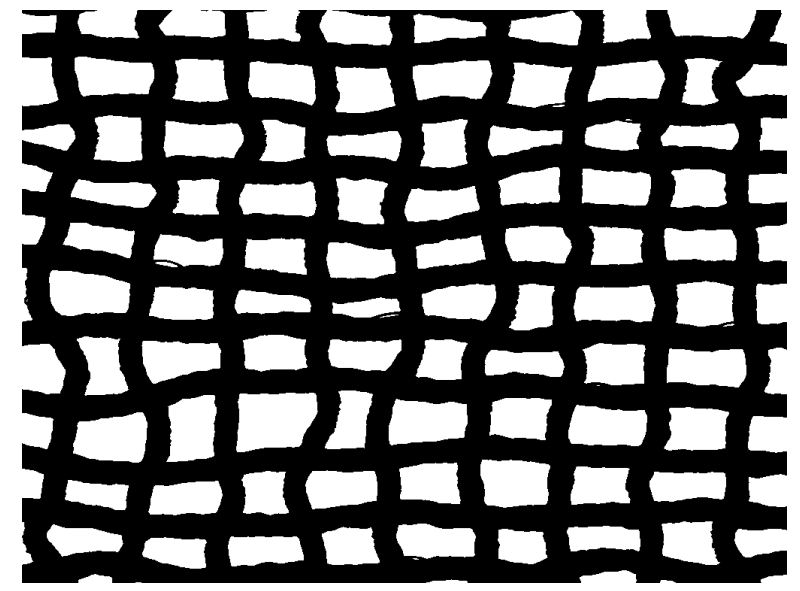

(d)

(e)

Figure 6 (a) Low CF fabric with a rectangle containing an integer number of periods. (b) PS of 6(a) and squared areas enclosing warp and weft frequency peaks to evaluate the centers of mass. (c) Intensity histogram of the image delimited by the rectangle in 6(a) displaying threshold values yielded by different thresholding methods. 1: MSSQ, 2: GM, 3: K-I, 4: Otsu, 5: K-means and 6: Visual. (d) Binarization of the cropped image by MSSQ method. (e) Binarization of the cropped image by Otsu’s method.

Several thresholding methods are applied to the cropped images. Tresholds found by Gaussian modeling $(G M)$, Kittler-Illingworth $(K-I)$, Otsu and K-means methods are compared to interactive visual thresholding (Visual) and the automatic method proposed in this paper (MSSQ) in figures 5(c) an 6(c). Differences among them should be evaluated from $C F$ 's yielded by those 
threshold values. Binarized images resulting from MSSQ threshold are shown in 5(d) and 6(d). Those from K-means threshold are displayed in 5(e) and 6(e). K-means and Otsu's thresholding methods provide coherent threshold values when they are applied to the low $C F$ sample, but they are too low for the high $C F$ sample. $K-I$ and $G M$ fail to provide consistent thresholding values for both samples. CF values corresponding to each method are found in tables 1 and 2. MSSQ thresholding method provides close values to Visual CF for both high and low CF samples.

\begin{tabular}{|l|r|l|}
\hline Method & \multicolumn{1}{|c|}{$L_{\mathrm{o}}$} & $C F$ \\
\hline$M S S Q$ & 150 & 0.935 \\
\hline$G M$ & 48 & 0.594 \\
\hline K-I & 86 & 0.828 \\
\hline Otsu & 92 & 0.847 \\
\hline K-means & 99 & 0.866 \\
\hline Visual & 151 & 0.937 \\
\hline
\end{tabular}

Table 1. Thresholds and CF yielded by different methods from the cropped image in 5(a).

\begin{tabular}{|l|r|l|}
\hline Method & $L_{0}$ & $C F$ \\
\hline$M S S Q$ & 133 & 0.603 \\
\hline$G M$ & 185 & 0.786 \\
\hline K-I & 9 & 0.483 \\
\hline Otsu & 95 & 0.589 \\
\hline K-means & 97 & 0.589 \\
\hline Visual & 93 & 0.586 \\
\hline
\end{tabular}

Table 2. Thresholds and CF yielded by different methods from the cropped image in 6(a).

Feature extraction from the digital image of a fabric sample and calculations yielding the final $C F$ value can be fully implemented in an algorithm. So the whole procedure is automatized and the $C F$ value directly obtained from the digital image.

\section{Validation of the Results}

The method has been applied to a set of 81 different plain fabric samples, resulting from the combination of four fabric features, whose effects on the $C F$ values are statistically analyzed in 
section 5. The features or factors considered are: type of fiber, warp yarn number, weft yarn number and weft thread count, each one investigated at three different levels. Thread counts in the warp direction were kept constant. Manufacturing specifications of these fabric samples are presented in table 3.

\begin{tabular}{|c|c|c|c|c|}
\hline \multicolumn{5}{|c|}{ Manufacturing Specifications } \\
\hline \multirow{2}{*}{ Fiber } & \multicolumn{2}{|l|}{ Yarn Number (tex) } & \multicolumn{2}{l|}{ Thread Count (yarns/cm) } \\
\cline { 2 - 5 } & Warp & Weft & Warp & Weft \\
\hline Cotton & $14,20,25$ & $14,20,25$ & 38 & $23,25,27$ \\
\hline Modal & $14,20,25$ & $14,20,25$ & 38 & $23,25,27$ \\
\hline Treated modal (modal sun) & $14,20,29$ & $14,20,29$ & 38 & $23,25,27$ \\
\hline
\end{tabular}

Table 3. Manufacturing specifications of the fabrics' samples.

$C F$ values obtained by the proposed method are compared to mean values of $C F$ evaluated according to an interactive thresholding procedure carried out by a group of experimented observers. We also analyze the relationship of the foregoing fabric features with $C F$ values yielded by the method.

Interactive visual thresholding is performed with the help of a PC. Two identical gray scale images of a sample are presented to the observer on a PC screen (Figure 7). The left side image remains fixed while the observer slides a cursor in order to select a threshold value. The pixels with intensities higher than the selected threshold change their color to magenta in the right side image for a better visualisation of the thresholding result. The observer sets the threshold value by seeking the best match between the bright pixels corresponding to the fabric holes in the left side image and the magenta pixels in the right side image. To conclude, $C F$ is straightforwardly obtained as the ratio of gray pixels to the total number of pixels of the right side image. 


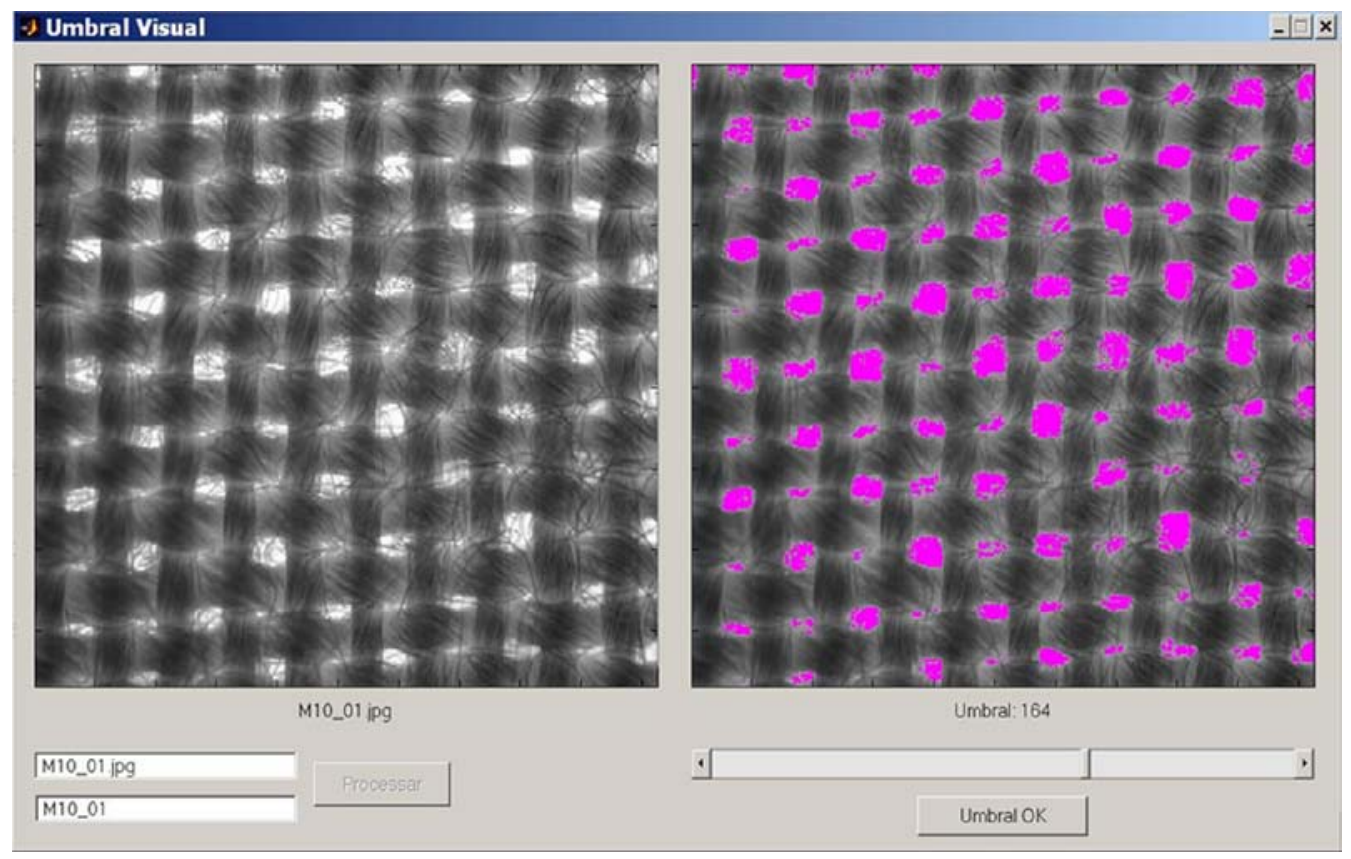

Figure 7 Screen presented to observers; left: fixed image; right: visually thresholded image.

Visual tresholding of the complete set of 81 samples has been performed by 20 different observers and the visual $C F$ value of each sample computed as the average of the 20 values individually obtained.

A correlation analysis to assess the relationship between $C F$ values obtained by the automatic method presented in this paper with both the visual $C F$ of the fabric samples and the type of fiber yields an overall determination coefficient of 94.71\%. Figure 8 displays three regression lines corresponding to the three types of fibers considered. All points are close to the diagonal. This fact indicates a very good agreement between the values obtained by both methods. The models fitted to modal and modal sun fibers are very similar and the model fitted to cotton fibers differs slightly from them. A possible explanation for that fact could be the higher irregularity of the cotton fiber when compared to modal and modal sun. The last fibers, although made from a natural polymer, have undergone a spinning process that provides a more uniform subsequent fabric surface, while cotton is a natural fiber and thus the cotton fabrics present more free ends of fibers arising from the surface. These free fibers on cotton fabrics would account for a higher 
difficulty to assess both the $C F$ values obtained by the proposed method and the visual $C F$. The confirmation of this conjecture, however, will require further research and analysis.

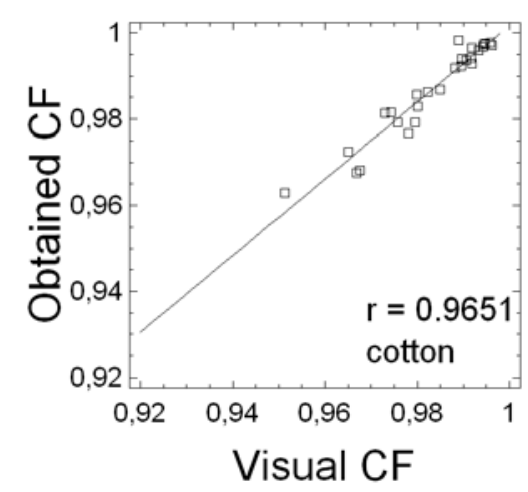

(a)

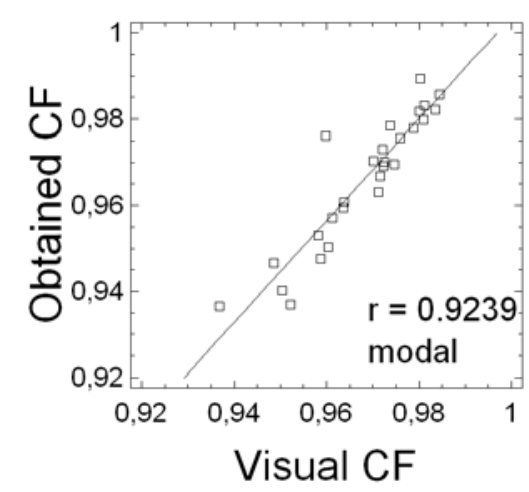

(b)

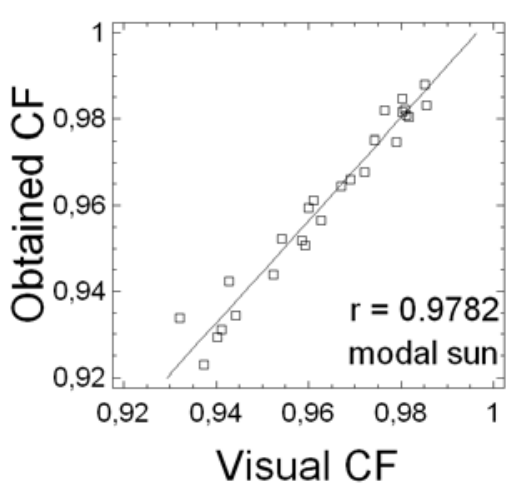

(c)

Figure 8 Comparison between visual $C F$ and $C F$ obtained by the method. Regression lines and linear correlation coefficients $r$ for the three types of yarns analyzed: a) Cotton b) Modal c) Modal sun.

The analysis of the relationship of the $C F$ obtained by the method presented in this paper with fabric features is performed by means of a multi-factor Analysis of Variance (ANOVA). Results are displayed in figure 9 and show a consistent behavior of the adjusted $C F$ mean values. An increase of yarn numbers for both warp and weft entails an increase of the adjusted mean $C F$ $(p<0.0001)$ as displayed in figures 9(a) and 9(b). Weft density values do not differ enough to produce a significant change on the adjusted mean $C F$ values $(p=0.6749)$ (Figure 9(c)). Fiber type also affects adjusted means $(p<0.0001)$ (Figure $9(d)$ ). 
Adjusted means according to warp yarn numbers

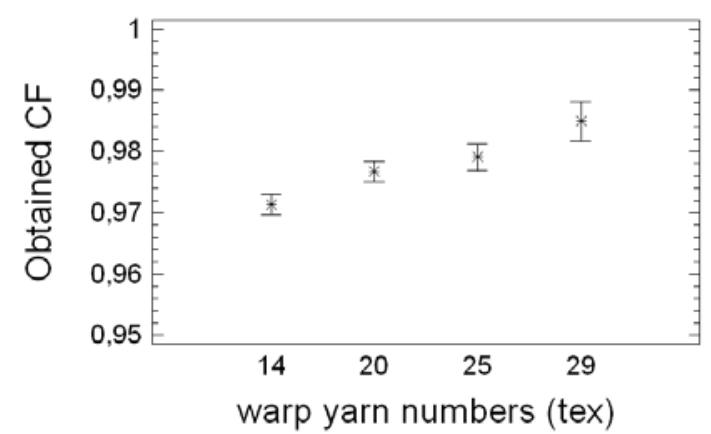

(a)

Adjusted means according to weft thread counts

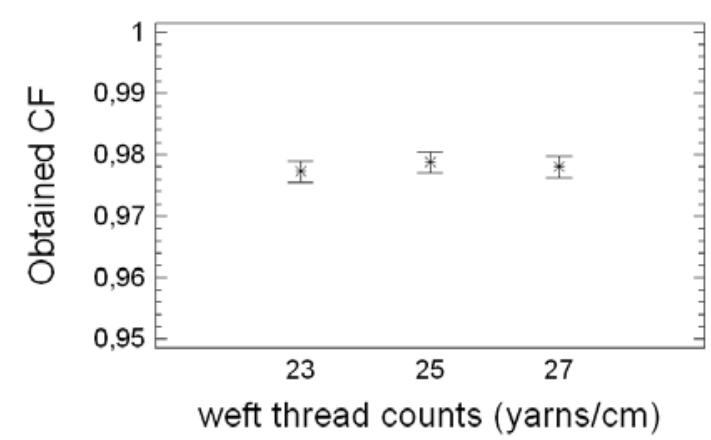

(c)
Adjusted means according to weft yarn numbers

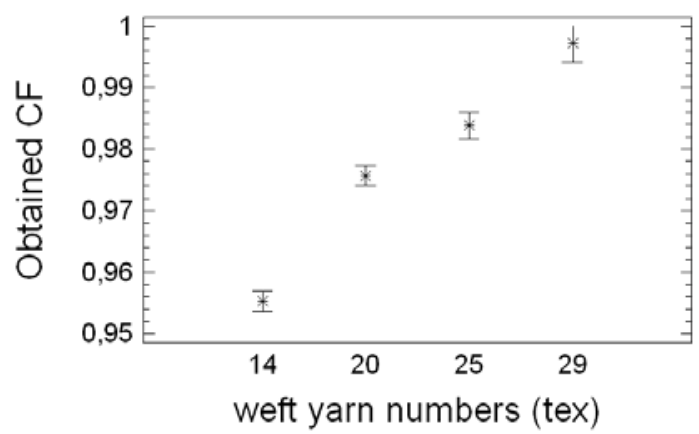

(b)

Adjusted means according to type of fiber

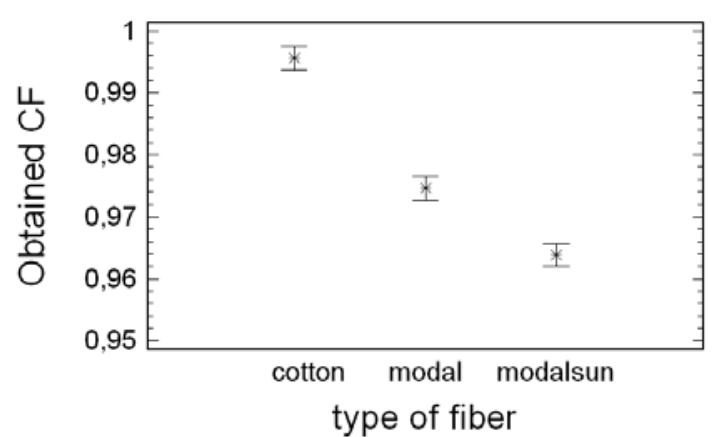

(d)

Figure 9 Multifactor ANOVA, relationship between the adjusted mean CF measured and: (a) Warp yarn numbers (b) Weft yarn numbers (c) Weft thread counts (d) Type of fiber.

\section{Conclusions}

We have developed an accurate, objective and automatic evaluation method of the CF of a fabric sample based on a single digital image of it, suitable for a wide range of $C F$ values, comprising values close to 1 . The algorithm is fully automatic and only requires as an input a digital image of the sample under inspection.

The method has been validated with a large set of plain fabric samples with known yarn numbers, thread counts and types of fiber. $C F$ values yielded by the method are consistent with fabric features. We have also found a very good agreement between these values and those obtained by an interactive visual thresholding of the images performed by a large set of observers, demonstrating its versatility and accuracy. 
Taking into account that fabric samples are illuminated by transmission together with the scale of the image field, the method should properly perform for interlacing patterns other than plain. All the mentioned characteristics show that this method can be very useful to implement quality control procedures to monitor CF of fabrics.

\section{Acknowledgements}

To Dr. Jordi Morató from Departament d’Òptica i Optometria of UPC, for supplying the system to capture the images. To MCYT (Proyecto DPI2006-05479) and FEDER for supporting this work.

\section{Literature Cited}

1. Marks, R., Lawton, P.J., Holme, D.A., An Introduction to Textiles. Vol. III - Fabrics, School of Textile Studies, Bolton Institute of Higher Education, UK (1993)

2. Milasius, V., Milasius, R., Kumpikaite, E., Olsauskiene, A., Influence of Fabric Structure on some Technological and End-use Properties, Fibres \& textiles in Eastern Europe, 11(2:41), 48-51 (2003)

3. Milasius, V., An Integrated Structure Factor for Woven Fabrics. Part I: Estimation of the Weave. Journal of the Textile Institute, 91 Part 1(2), 268-276 (2000)

4. Seyam, A., El-Shiekh, A., Mechanics of Woven Fabrics. Part III: Critical Review of Weavability Limit Studies, Textile Research Journal, 63(7), 371-378 (1993)

5. Seyam, A., El-Shiekh, A., Mechanics of Woven Fabrics. Part IV: Critical Review of Fabric Degree of Tightness and Its Applications, Textile Research Journal, 64(11), 653-662 (1994)

6. Olsauskiene, A., Milasius, R., Dependence of Air Permeability on Various Integrated Fabric Firmness Factors, Materials Science, 9(4), 401-404 (2003) 
7. Algaba, I., Riva, A., Crews, P.C., Influence of Fiber Type and Fabric Porosity on the UPF of Summer Fabrics, AATCC Review, 4(2), 26-31 (2004)

8. Peirce, F.T., The Geometry of Cloth Structure, Journal of the Textile Institute, 28, 45-96 (1937)

9. Brierley, S., Cloth Settings Reconsidered, The Textile Manufacturer, 78, 349-351; 79, 533537; 595-597 (1952)

10. Galcerán, V., Tecnología del tejido. Vol. 2: Análisis de tejidos y técnica de su fabricación. $2^{\mathrm{a}}$ Ed, ETSIIT, Terrassa (1961)

11. Galuszynski, S., Structure and Tightness of Woven Fabrics, Indian Journal of Textile Research, 12, 71-77 (1987)

12. Castellar, M.D., Manich, A.M., Carvalho, J., Barella, A., An application of the image analysis to the woven fabric cover factor determination, in "Niches in the World of Textiles: The 77th World Conference of the Textile Institute, Tampere”, Vol. 2, 56-72, The Textile Institute, Manchester (1997)

13. Kang, T.J., Choi, S.H., Kim, S.M., Oh, K.W., Automatic Structure Analysis and Objective Evaluation of Woven Fabric Using Image Analysis, Textile Research Journal, 71(3), 261$270(2001)$

14. Abou-llana, M., Youssef, S., Pastore, C., Gowayed, Y., Assessing Structural Changes in Knits During Processing, Textile Research Journal, 73(6), 535-540 (2003)

15. Cardamone, J.M., Damert, W.C., Phillips, J.C., Marmer, W.N., Digital Image Analysis for Fabric Assessment, Textile Research Journal, 72(10), 906-916 (2002)

16. Sezgin, M., Sankur, B., Survey over image thresholding techniques and quantitative performance evaluation, Journal of Electronic Imaging, 13 (1), 146-168, (2004).

17. Gonzalez, R.C., Woods, R.E., Digital Image Processing, Adisson-Wesley, London (1993) 
18. Xu, B., Identifying Fabric Structures with Fast Fourier Transform Techniques, Textile Research Journal, 66 (8), 496-506(1996)

19. Escofet, J., Millán, M.S., Ralló, M., Modelling of woven fabric structures based on Fourier image analysis, Applied Optics, 40(34), pp. 6170-6176 (2001)

20. Ralló, M., Escofet, J., Millán, M.S., Weave-repeat identification by structural analysis of fabric images, Applied Optics, 42(17), pp. 3361-3372 (2003)

21. Russ, J.C., The Image Processing Handbook. $2^{\text {nd }}$ ed, CRC Press, Boca Raton FL (1995)

22. Koren, N., Making fine prints in your digital darkroom, available at: http://www.normankoren.com/makingfineprints1A.html (accessed 5 September 2008) (2000)

23. Holst, G.C., CCD arrays, cameras and displays, SPIE Optical Engineering Press, Bellingham (WA) (1998) 